\title{
Floristic Study and Species Diverisy of Msallata-Garaboulli Province in Libya
}

\author{
Abdurrazag S. Sherif ${ }^{1}$, Mohammed H. Mahklouf ${ }^{2}$, Abdalla G. Betelmal ${ }^{1}$ \\ ${ }^{1}$ Natural Reassures Department, Faculty of Agriculture, University of Tripoli, Libya \\ ${ }^{2}$ Botany Department, Faculty of Sciences, University of Tripoli, Libya \\ E-mail: Shabda4418@yahoo.com
}

\begin{abstract}
:
A survey of plant species of Msallata and Garaboulli was taken in between 2017-2018. A total number of 468 different plant taxa have been collected from the study area representing 68 families of which 57 families and 389 species are belonging to dicotyledons, 8 families and 76 species belonging to monocotyledons, and 3 families with one species each belonging to Gymnosperms. The results of this study shows that the dominance of the family Asteraceae with 78 species followed by the family Fabaceae with 59 species, the family Poaceae with 47 species, Brassicaceae with 27 species and Apiaceaew with 19 species. Other families such as Liliaceae, Caryophylaceae, Lamiaceae, Cistaceae, Boraginaceae, Plantaginaceae, and Rubiaceae were represented by 16, 15, 14, 13, 12, 10, 10 species respectively. The result have also shown that the gerera Plantago, and Silene are the most sizable genera with 10, 8 species respectively. Lifeform spectrum analysis have shown the predominance of therophytes with 231 species, followed by Hemicryptophytes with 62 species, while chorotype spectrum analysis have shown the dominance of Mediterranian species, followed by Mediterrean/Iranu-Turanean species.
\end{abstract}

Keywords:

flora; floristic; plant diversity; msallata; gharaboulli

\section{Introduction}

Libya is a country in the North African region. It lies along the southern coast of the Mediterranean, approximately between latitude $18^{\circ}$ and $33^{\circ}$ North and $9^{\circ}$ and $25^{\circ}$ East (Figure 1) and occupies an area of about 1, 759, 540 square kilometres (El-Mokasabi, 2017), of which more than $90 \%$ of it is desert with exception of the coastal strip, Al Jabal ElAkhdar, and Jabal Nafousa regions (El-Darier, \& El-Mogaspi, 2009). Boulos (1972) indicated that the coastal belt, which extends from the Tunisian to the Egyptian borders, is about 5.2\% of the whole country. This area is quite fertile and receives an adequate amount of rainfall in winter, particularly in the east and west, thus a great part of this belt exhibits the typical Mediterranean flora. The climate is typical of the Mediterranean, characterized by the cool, rainy winter and hot dry summer. Whereas, the over most climate of the country being hot, arid Sahara, but it is moderated along the coastal littoral by the Mediterranean Sea Al-Sghair, et al, 2019).

The vascular flora of Libya has appeared in publications by different authores, which included Boulus (1972) who listed 1600 plant speciec of flowering plants, whereas; Qaiser and El-Gadi. (1984) reported 1750 vascular plant species. Publications of the Flora of Libya by Jafri, S. M. H. and El-Gadi (1976-1990) revealed the presence of 2028 plant species representing 779 genera, and 149 families of Angiospermis. Sherif and Ben-Othman. (1992) reported 2059 vascular plant species (excluding Pteridophytes) belonging to 787 genera, and 155 families. While, Feng et al (2013) reported 2103 vascular plant species that belong to 856 genera and 155 families. Of which seed plants were charactherized by highest number of herbs (annual to perennial), and low number of woody (tree and shrub) species; these have an 
important influence on the structure of floral composition, the geographic element of the flora was predominantly tropical and Mediterranean (Mahklouf \& Al-Sghair, 2016). The floristic composition of plants in Libya is still comparatively unknown as far as in-depth ecological and botanical studies (Pergent et al, 2002).

The history of plant exploration in Libya has become the interest of many workers. For example, the most comprehensive floristic studies in Libya was presented as a preliminary checklist of the flora of Libya by Keith (1965), and Flora of Libya by Jafri and El-Ghadi (1977 - 1990), furthermore. In addation to that, there were a few regional floristic studies on Msallata district such as biodiversity of the Msallata national reserve (Bashir and Erteeb, 2007), and flora of Wadi Gerreem (Al-Osta and Erteeb, 2018). Since the flora of Msallata and ElGaraboulli has not been studied thoroughly during the work on the flora of Libya (19761989). Therefore, the purpose of this survey is to have an exclusive study to its flora.

\section{Review of Literatures}

This paper deals mainly with the flora of Msallata and El-Garaboulli Districts, which is located about $60 \mathrm{~km}$., east of Tripoli (Capital) and occupies between. (34॰ 32' 58.87' N, 02。 14 ' 20.89" E), and it is ranges between $100-500 \mathrm{~m}$ above the see level as measured by GPS. The study area is bounded by the sea to the north, El-Gweaa to the west, Al-koms to the east, and Tarnuna to the south (Figure 1). The climate of the study area followes the climate of the Mediterranean region, which is cold \& rainy at the winter with an averge rainfall, ranges between 100-300 mm annually, and hot \& dry at the summer with a mean of $18^{\circ} \mathrm{C}($ Feng et al, 2013). Climate is one of the most important factors affecting biodiversity, vegetation distribution, and soil composition, and the high temperature affects vegetation and the dominant species (Alftisi et al, 2019).

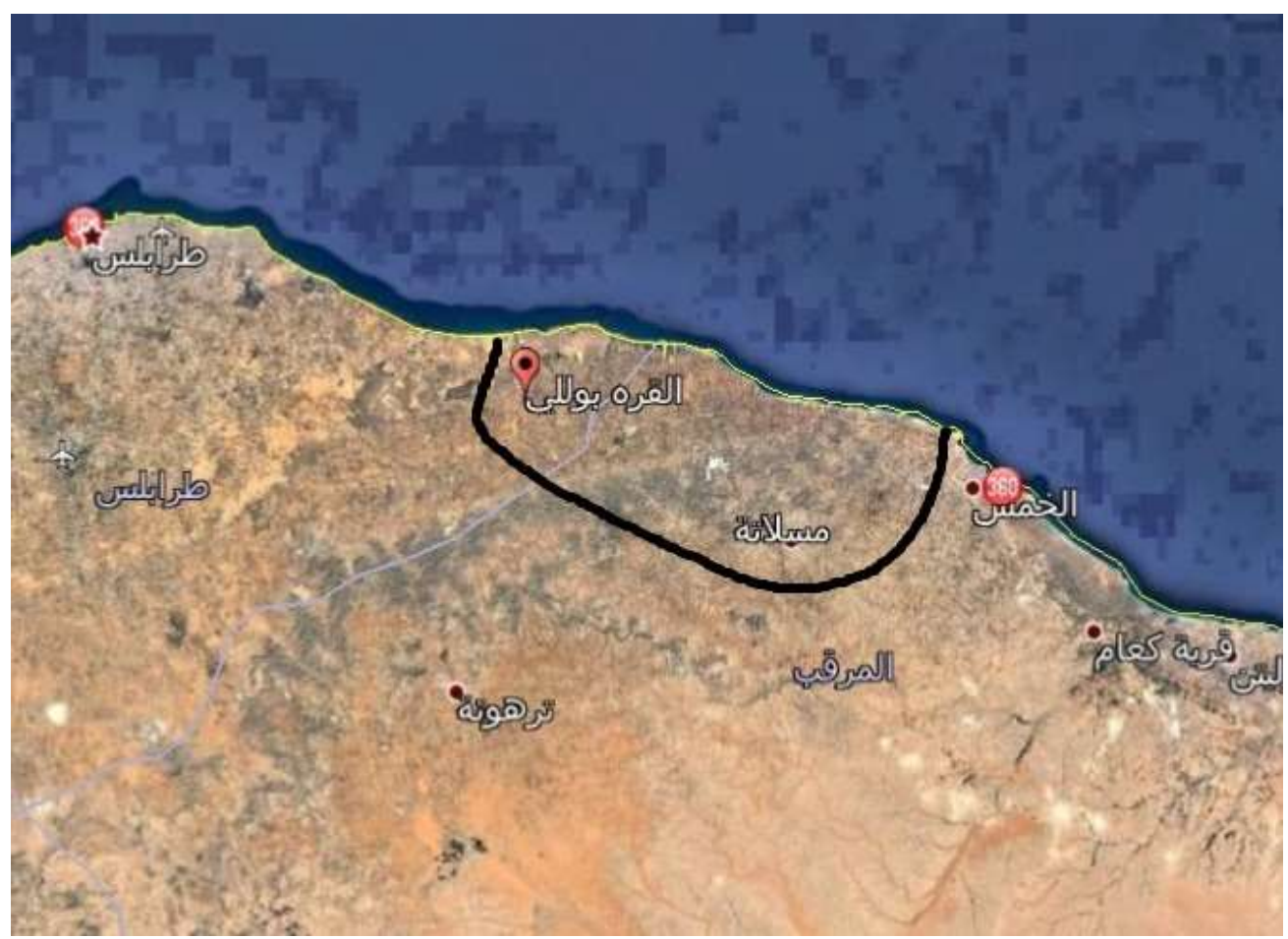

Figure 1. Shows the study area 


\section{Research Methods}

A total number of 468 plant specimens were collected in between 2017-2018 upon various field trips. The collected plants were then treated by the usual herbarium procedures including pressing, poisoning, mounting, labeling, and identifying. Collection and Identification of plant species was done by the authors with the aid of the following litheratures (Keith, 1965; Jafri and El-Ghadi, 1977 - 1990; Sherif and El-Taife, 1986). Eventually, the identified plant specimens were deposited at the national herbarium, Botany Department, Faculty of Sciences, Tripoli University.

\section{Discussion}

The flora of Msallata and Garaboulli represented by 468 different plant taxa belonging to 68 families, 247 genera, and 468 species. Three different plant groups gymnosperms with 3 families and 3 species, dicotyledones with 57 families and 389 species, and monocotyledons with 8 families and 76 species (Appendix). The families Asteraceae, Fabaceae, Poaceae, and Brassicaceae are considered as the most dominant and sizable families with 76, 59, 47 and 27 plant species respectively (Table 1 \& figue 2). Other families such as Apiacaceae, Liliaceae, Caryophyllaceae, Lamiaceae, and Cictaceae are less dominant and represented by 19, 16, 15, 14 \& 13 species respectively. Whereas, the rest of the families are represented by 12 species or less. The results of this study shows that the most dominant genera are plantago with 10 species, and Silene with 8 specie. Whilst, genera such as Medicago, Erodium, Euphorbia, Helianthemum and Centaurea are represented by 7 species each. Genera such as Astragalus, Ononis, Convolvulus and Bupleurum represented by 6 species each.While, the rest of the genera are represented by 5 species or less (Table $2 \&$ figure 3 ).

Table 1. Shows dominant families.

\begin{tabular}{|l|c|}
\hline \multicolumn{1}{|c|}{ Family } & No of species \\
\hline Asteraceae & 76 \\
\hline Fabaceae & 59 \\
\hline Poaceae & 47 \\
\hline Brassicaceae & 27 \\
\hline Apiaceae & 19 \\
\hline Liliaceae & 16 \\
\hline Cryophyllaceae & 15 \\
\hline Lamiaceae & 14 \\
\hline Cistaceae & 13 \\
\hline Boraginaceae & 12 \\
\hline Plantaginaceae & 10 \\
\hline Rubiaceae & 10 \\
\hline
\end{tabular}




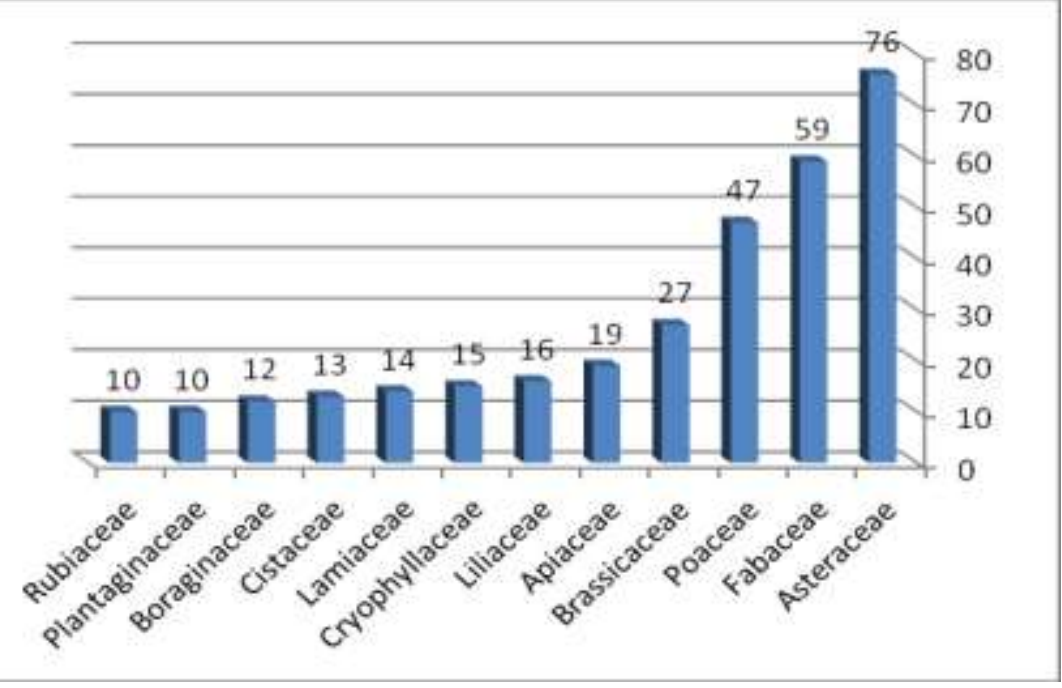

Figure 2. Shows dominant families

Table 2. Shows dominant genera.

\begin{tabular}{|c|c|}
\hline Genus & No of species \\
\hline Plantago & 10 \\
\hline Silene & 8 \\
\hline Medicago & 7 \\
\hline Helianthemum & 7 \\
\hline Euphorbia & 7 \\
\hline Erodium & 7 \\
\hline Centaurea & 7 \\
\hline Astragalus & 6 \\
\hline Ononis & 6 \\
\hline Convolvulus & 6 \\
\hline Buplaurum & 6 \\
\hline Trifolium & 5 \\
\hline Gallium & 5 \\
\hline Bromus & 5 \\
\hline
\end{tabular}

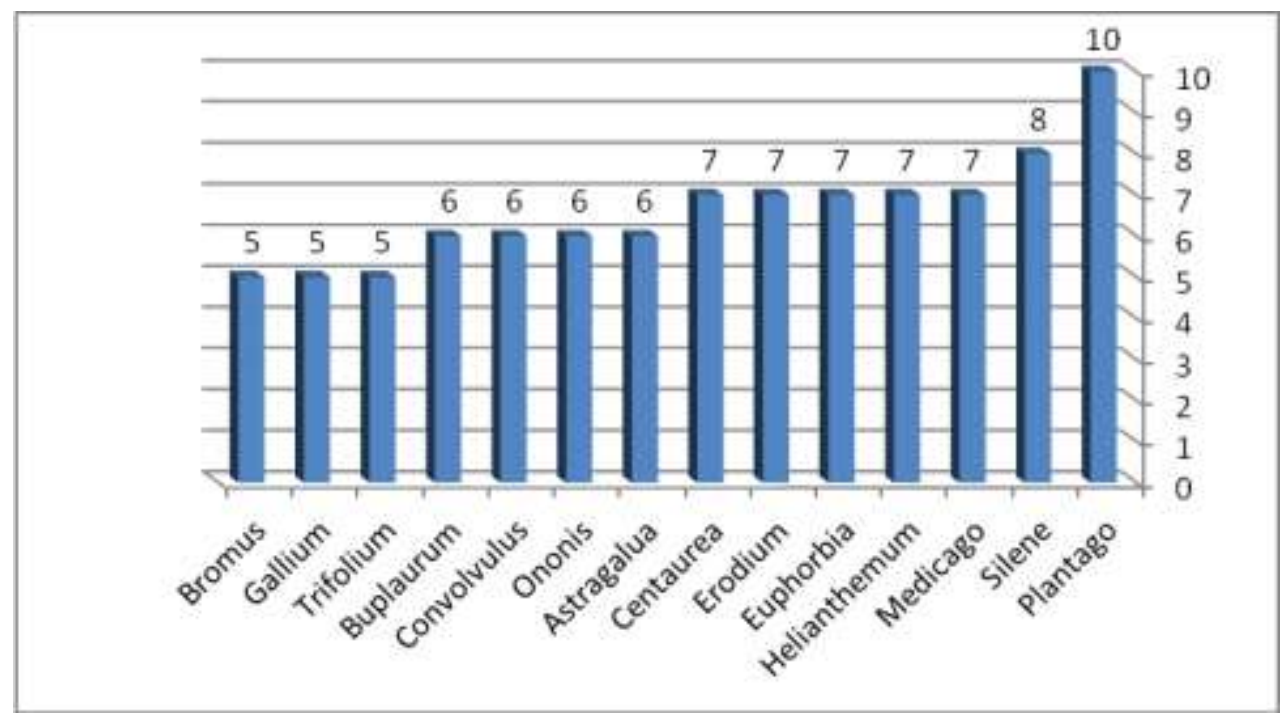

Figure 3. Shows dominant genera. 
Life form spectrurm of species was analyzed according to Raunkiae system (1934) as modified by Govaerts et al.(2000) . Such system showed absolute dominance of Therophytes with 302 species, followed by Hemicryptophytes with 62 species, and Geophytes with 44 species, the rest of life forms were less frequent, that Chaemephytes with 27 species, Nanophanerophytes with 23 species, and Phanerophytes with 11 species (Tables 3 and Appendix) (Fig 4).

Table 3. Shows lifeforms of different species.

\begin{tabular}{|l|c|l|}
\hline \multicolumn{1}{|c|}{ Lifeform } & No of species & \multicolumn{1}{c|}{$\mathbf{~}$} \\
\hline Therophytes & 302 & 65.5 \\
\hline Hemicryptophtes & 62 & 13.25 \\
\hline Geophytes & 44 & 9.4 \\
\hline Chaemephytes & 27 & 5.5 \\
\hline Nanophanerophytes & 23 & 4.9 \\
\hline Phanerophytes & 11 & 2.35 \\
\hline
\end{tabular}

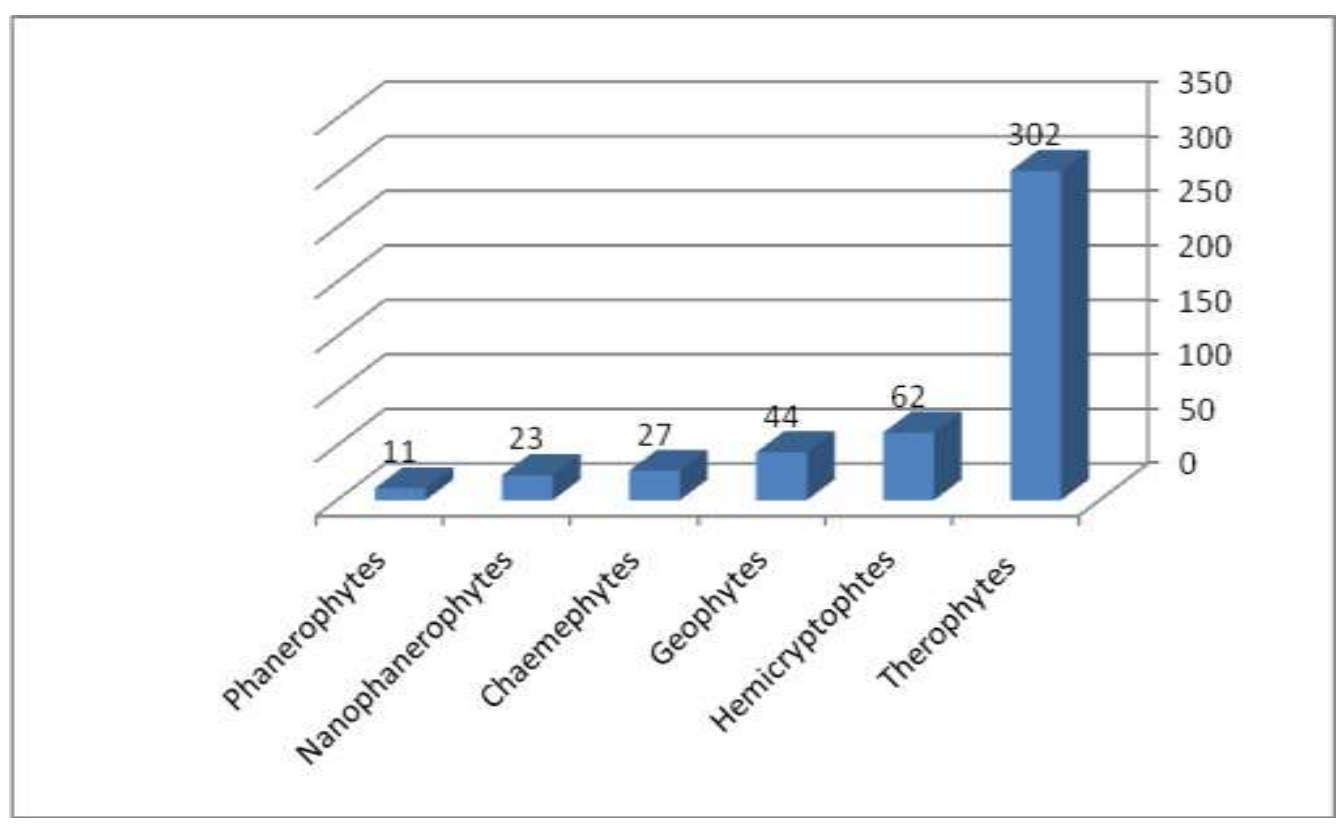

Figure 4. Shows lifeforms of different species.

Chorological spectrum of collected and identified plant species were also analyzed. The results have shown absolute predominance of Mediterranean species with 231 species, followed by Med./ Ir-Tu. species with 101 species, Med./ Ir-Tu./ Eur-Si species with 27 species, and Pluriregionsl species with 26 species, the rest of chorological spectra were with little frequent as shown in (Tables 4 and Appendix) (Fig 5).

Table 4. Shows number of species and their percentage in chorotypes.

\begin{tabular}{|l|c|l|}
\hline \multicolumn{1}{|c|}{ Chorotype } & No of species & \multicolumn{1}{c|}{$\%$} \\
\hline Med & 231 & 49.3 \\
\hline Med./ Ir-Tu. & 101 & 21.6 \\
\hline Med./ Ir-Tu./ Eur-Si. & 27 & 5.8 \\
\hline Plu & 26 & 5.5 \\
\hline Med./ Eur-Si. & 22 & 4.7 \\
\hline Sah-Ar. & 20 & 4.3 \\
\hline Med./ Sah-Ar. & 6 & 1.3 \\
\hline
\end{tabular}




\begin{tabular}{|l|l|l|}
\hline Cos & 5 & 1.0 \\
\hline Ir-Tu./ Sah-Ar. & 4 & 0.9 \\
\hline Ir-Tu & 3 & 0.7 \\
\hline
\end{tabular}

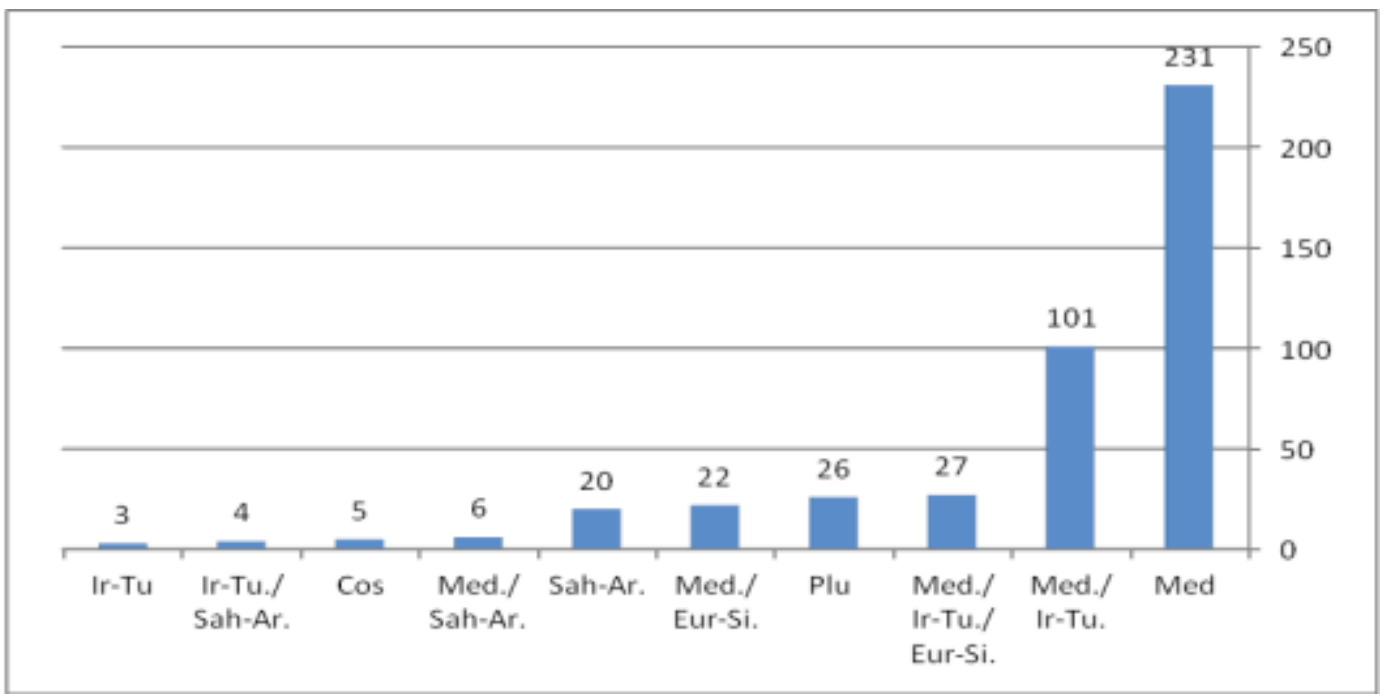

Figure 5. Shows number of species in chorotypes

The dominance of the familes Asteraceae, Fabaceae, Poaceae was expected because such families dominated the Mediterranean climate. In addation to that, these families are cosmopolitan in distribution. Moreover, the dominance of Therophytes and Mediterranean chorotypes agreed with our expectations since the study area falls within the coastal Mediterranean region. The results have also been revealed that the most characteristic features of the flora of Msallata \& Garaboulli is that the large number of families recorded in this study, wchic is close to the half number of the total families in the flora of Libya, this findings indicates that the flora of Msallata-Gharaboulli is rich.

\section{Conclusion}

The present study is the first research to investigate the floral divesity of the study area, which is located about $60 \mathrm{~km}$., east of Tripoli. The study revealed presence of 468 different plant species representing 68 families. The family Asteraceae was the most dominant with 78 species followed by the family Fabaceae with 59 species, the family Poaceae with 47 species, Brassicaceae with 27 species and Apiaceaew with 19 species, The result have also shown that the gerera Plantago, and Silene are the most sizable genera with 10, 8 species respectively. Lifeform spectrum analysis have shown the predominance of therophytes with 231 species, while chorotype spectrum analysis have shown the dominance of Mediterranian species.

\section{References}

Alftisi1, M; Osman , R; Elalem1, R; Al-Sghair, F. 2019. Ecological Characteristics Study of the Vegetation Cover for the Protected Area Faculty of Agriculture at the University of Tripoli-Libya. Budapest International Research in Exact Sciences (BirEx) Journal. 1(4). 62-69.

Al-Osta, S. M; Erteeb, F. B. 2018. Study of Vascular Flora of Wadi Gerream in Msallata, MSc, University of Tripoli. Faculty of Sciences. Deparment of Botany. Tripoli, Libya. 
Al-Sghair, F. G; Mahklouf, M. H; Abudaya, E. A. 2019. Species Diversity and Floristic Analysis of the Family Poaceae in Libya Depending on the Flora of Libya. Advances in Bioscience and Bioengineering. 7(2): 13-21. doi: 10.11648/j.abb.20190702.11

Bashir, S; Erteeb, F. 2007. Systematic study of Msallata National Reserve. AlFaateh.University. Faculty of Sciences. Deparment of Botany. Tripoli, Libya.

Boulos, L. 1972. Our present knowledge on the Flora and Vegetation of Libya. Bibliography. Webbia, 26 (11), 365-400.

El-Darier, S. M \& El-Mogaspi, F. M. (2009). Ethnobotany and relative importance of some endemic plant species at El-Jabal El-Akhdar region (Libya). World J. of Agric. Sci. 5 (3),353-360.

El-Mokasabi, F. M. 2017. Studies on the Flora of Libya [Version 1; awaiting peer review]. ContROL 1: 08. doi: 10.28915/control.0008.1.

Feng Y, Lei JQ, Xu XW. 2013. Composition and Characteristics of Libyan Flora. Biol Sci Belgrade. 65(2): 651-7.

Pergent G, Djellouli A, Hamza AA, Ettayeb KS, El Mansouri AA, Talha FM. 2002. Characterization of the benthic vegetation in the Farwà Lagoon (Libya). J Coastal Conserv. 8(2):119-26. DOI: 10.1652/1400-0350(2002)008[0119:COTBVI]2.0.CO;2

Keith, H. G. 1965. A preliminary checklist of Libyan flora. London: Government of the Libyan Arab Republic, Ministry of Agriculture and Agrarian Reform. Vol, 1 \& II. .

Jafri, S. M; El - Gadi, A. A. 1976-1990. Flora of Libya, Al-Faateh.University. Faculty of Sciences. Deparment of Botany. Tripoli, Libya.

Govaerts, R; Frodin, D. G; Radcliffe-Smith, A. 2000. World Checklist and Bibliography of Euphorbiaceae (with Pandanaceae). Kew: The Royal Botanic Gardens.

Qaiser, M. \& El-Gadi, 1984. A. A critical Analysis of the Flora of Libya. The Libyan Journal of Science 13: 31-40.

Raunkiaer, C. 1934. The Life Forms of Plants and Statistical Plant Geography. Oxford: Th Clarendon Press.

Sherif, A. S. \& Ben-Othman, A. R. 1992. Checklist Analysis of El-Naser Forest Flora, "Tripolitania". Bull. Nat. Herb. Trip. Tripoli-Libya, 3: 9-20.

Sherif, A.S. and El-Taife, A. 1986. Flora of Libya, Gymnosperms, Fac. Sci. Dept. Bot.,AlFaateh University, Tripoli.

Appendix

\begin{tabular}{|c|c|c|c|c|}
\hline No & Family & Species & Lifeform & Chorotype \\
\hline \multicolumn{5}{|r|}{ Gymnosperms } \\
\hline 1 & Cupressaceae & Juniperus phoenicea L. & $\mathrm{Ph}$ & Med. \\
\hline 2 & Ephedraceae & Ephedra altissima Desf. & $\mathrm{NP}$ & Med. \\
\hline 3 & Pinaceae & Pinus halipensis L. & $\mathrm{Ph}$ & Med. \\
\hline \multicolumn{5}{|r|}{ Monocotyledons } \\
\hline 4 & Alliaceae & Allium ampeloprasum L. & Geo & Med. \\
\hline 5 & " & $\begin{array}{r}\text { Allium negrianum Maire \& } \\
\text { Weiller }\end{array}$ & Geo & Med. \\
\hline 6 & $"$ & Allium nigrum $\mathrm{L}$. & Geo & Med. \\
\hline 7 & $"$ & Alliumleucanthum C. Koch in L. & Geo & Med. \\
\hline 8 & Amaryllidaceae & Pancartium maritimum L. & Geo & Med. \\
\hline 9 & " & Pancratium foetidum Pomel. & Geo & Med. \\
\hline 10 & Araceae & Arisarum vulgare Targ. Tozz & Geo & Med. \\
\hline 11 & Cyperaceae & Scirpus holoschoenus L. & Geo & Med./ Ir-Tu. \\
\hline 12 & Iridaceae & Gladiolus byzantinus Miller. & Geo & Med. \\
\hline 13 & $"$ & Iris planifolia (Mill.) Durand \& & Geo & Med./ Ir-Tu. \\
\hline
\end{tabular}




\begin{tabular}{|c|c|c|c|c|}
\hline & & Barratte & & \\
\hline 14 & $"$ & Iris sisyrinchium $\mathrm{L}$. & Geo & Med. \\
\hline 15 & Liliaceae & $\begin{array}{r}\text { Androcymbium gramineum (Cav.) } \\
\text { Mc Brid }\end{array}$ & Geo & Med. \\
\hline 16 & $"$ & Asparagus aphyllus L. & Geo & Med. \\
\hline 17 & $"$ & Asparagus stipularis Forsk. & Geo & Med. \\
\hline 18 & $"$ & Asphodelus aestivus Brot. & Geo & Med. \\
\hline 19 & $"$ & Asphodelus fistulosus L. & Geo & Med. \\
\hline 20 & $\bar{~}$ & $\begin{array}{r}\text { Asphodelus microcarpus Salzm. \& } \\
\text { Viv. }\end{array}$ & Geo & Med. \\
\hline 21 & $"$ & Bellevalia sessiliflora (Viv.)Kunth & Geo & Med. \\
\hline 22 & $"$ & Dipcadi serotinum (L.) Medic. & Geo & Plu. \\
\hline 23 & $"$ & Gagea fibrosa (Desf.) Schult. & Geo & Med. \\
\hline 24 & $"$ & Muscari comosum (L.) Mill. & Geo & Med. \\
\hline 25 & $"$ & Muscari racemosum (L.) Mill. & Geo & Med. \\
\hline 26 & $"$ & Ornithogalum arabicum $\mathrm{L}$ & Geo & Med. \\
\hline 27 & $\bar{~}$ & Ornithogalum pyrenaicum L. & Geo & $\begin{array}{l}\text { Med./ Ir-Tu./ } \\
\text { Eur-Si. }\end{array}$ \\
\hline 28 & $"$ & Scilla peruviana $\mathrm{L}$. & Geo & Med. \\
\hline 29 & $"$ & Urginea autumnalis $\mathrm{L}$. & Geo & Med. \\
\hline 30 & " & Urginea maritima (L.) Baker & Geo & Med. \\
\hline 31 & Orchidaceae & Ophrys speculum Link. & Geo & Med. \\
\hline 32 & $"$ & Orchis coriophora L. & Geo & Med./ Ir-Tu. \\
\hline 33 & Poaceae & Aegilops geniculata Roth. & $\mathrm{Th}$ & Med./ Ir-Tu. \\
\hline 34 & $"$ & Aegilops Kotschyi Boiss. & $\mathrm{Th}$ & Med./ Ir-Tu. \\
\hline 35 & $"$ & Aristida adscensionis L. & $\mathrm{Th}$ & Med. \\
\hline 36 & $"$ & Avellinia mitchelii & $\mathrm{Th}$ & Med. \\
\hline 37 & $"$ & Avena barbata Pott. ex Link. & Th & Med./ Ir-Tu. \\
\hline 38 & $"$ & Avena sterilis $\mathrm{L}$. & $\mathrm{Th}$ & Med./ Ir-Tu. \\
\hline 39 & $"$ & Briza maxima L. & $\mathrm{Th}$ & Med. \\
\hline 40 & $"$ & Bromus diandrus Roth. & $\mathrm{Th}$ & Med. \\
\hline 41 & 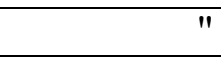 & Bromus madritensis $\mathrm{L}$. & $\mathrm{Th}$ & Plu. \\
\hline 42 & $"$ & Bromus molliformis Lloyd. & $\mathrm{Th}$ & Med./ Eur-Si. \\
\hline 43 & $"$ & Bromus rigidus Roth. & $\mathrm{Th}$ & Med./ Eur-Si \\
\hline 44 & $\bar{~}$ & Bromus rubens $\mathrm{L}$. & $\mathrm{Th}$ & $\begin{array}{l}\text { Med./ Ir-Tu./ } \\
\text { Eur-Si. }\end{array}$ \\
\hline 45 & $"$ & Catapodium marinum $\mathrm{L}$. & Th & Med./ Eur-Si. \\
\hline 46 & $"$ & Cenchrus ciliaris $\mathrm{L}$. & $\mathrm{Th}$ & Sah-Ar. \\
\hline 47 & " & $\begin{array}{r}\text { Cutandia dichotoma (Forsk) } \\
\text { Trabut. }\end{array}$ & $\mathrm{Th}$ & Med./ Ir-Tu. \\
\hline 48 & $"$ & Cutandia maririma (L.) Barbey & $\mathrm{Th}$ & Med. \\
\hline 49 & $"$ & Cynodon dactylon (L.) Pers. & Geo & Boreal. Trop. \\
\hline 50 & $\bar{"}$ & $\begin{array}{r}\text { Cynosurus coloratus Lehm. ex } \\
\text { Steud. }\end{array}$ & $\mathrm{Th}$ & Med. \\
\hline 51 & $"$ & Cynosurus elegans Desf. & Th & Med./ Ir-Tu. \\
\hline 52 & $"$ & Dactylis glomerata L. & Th & Med./ Ir-Tu. \\
\hline 53 & $"$ & $\begin{array}{r}\text { Gastridium ventricosum (Gouan.) } \\
\text { Schin et Thell. }\end{array}$ & $\mathrm{Th}$ & Med./ Ir-Tu. \\
\hline 54 & $"$ & Hordeum murinum L. & $\mathrm{Th}$ & Plu. \\
\hline 55 & $"$ & Hordeum spontaneum C. Koch. & $\mathrm{Th}$ & Med./ Ir-Tu. \\
\hline
\end{tabular}




\begin{tabular}{|c|c|c|c|c|}
\hline 56 & $"$ & Hyparrhenia birta (L.) Stapf & $\mathrm{H}$ & Plu. \\
\hline 57 & " & $\begin{array}{r}\text { Imperata cylindrica (L.) } \\
\text { Reauschel. }\end{array}$ & Geo & Med./ Ir-Tu. \\
\hline 58 & $"$ & Lagurus ovatus L. & Th & Plu. \\
\hline 59 & $"$ & Lamarckia aurea (L.) Moench & Th & Med./ Ir-Tu./Sud \\
\hline 60 & $"$ & Lolium loliaceum Bory \& Chaub. & Th & Med./ Ir-Tu. \\
\hline 61 & $"$ & Lolium multiflorum Lam. & Th & Med./ Eur-Si. \\
\hline 62 & $"$ & Lolium rigidum Gaud. & Th & Plu. \\
\hline 63 & " & $\begin{array}{r}\text { Lophochloa salzmannii Boiss } \\
\& \mathrm{H} . \text { scholz }\end{array}$ & Th & Med. \\
\hline 64 & $"$ & Lygeum spartum Loefl. ex L. & Geo & Med. \\
\hline 65 & " & $\begin{array}{r}\text { Parapholis incurve (L.) C.E. } \\
\text { Hubbard }\end{array}$ & Th & $\begin{array}{l}\text { Med./ Ir-Tu./ } \\
\text { Eur-Si }\end{array}$ \\
\hline 66 & " & $\begin{array}{r}\text { Pennisetum divisum (Forsk. ex } \\
\text { Gmel.) Hem. }\end{array}$ & Geo & Sah-Ar. \\
\hline 67 & " & $\begin{array}{r}\text { Pennisetum setaceum (Forsk.) } \\
\text { Chiov. }\end{array}$ & Geo & $\begin{array}{l}\text { Med./ Ir-Tu./ } \\
\text { Sud. }\end{array}$ \\
\hline 68 & $"$ & Phalaris minor Retz. & Th & Med./ Ir-Tu. \\
\hline 69 & " & $\begin{array}{r}\text { Phragmites australis (Cav.) Trin. } \\
\text { ex steud. }\end{array}$ & Geo & Cos. \\
\hline 70 & $"$ & Piptatherum miliaceum (L.) Coss. & $\mathrm{H}$ & Med. \\
\hline 71 & $"$ & Poa annua $\mathrm{L}$. & Th & Plu. \\
\hline 72 & $"$ & Poa sinaica $\mathrm{L}$. & $\mathrm{H}$ & Ir-Tu. \\
\hline 73 & $"$ & Polypogon monspeliensis (L) Desf. & Th & Plu. \\
\hline 74 & $"$ & Psilurus incurvus Gouan. & Th & Med./ Ir-Tu. \\
\hline 75 & $"$ & Stipa barbata Desf. & Geo & Med./ Ir-Tu. \\
\hline 76 & $"$ & Stipa capensis Thunb. & Th & $\begin{array}{r}\text { Med./ Ir-Tu./ } \\
\text { Sah-Ar. }\end{array}$ \\
\hline 77 & $"$ & Stipa parviflora Desf. & Geo & Med./ Ir-Tu. \\
\hline 78 & $"$ & Stipa tenacissima $\mathrm{L}$. & Geo & Med. \\
\hline 79 & $"$ & Trachynia distachya (L.) Link. & Th & Med./ Ir-Tu. \\
\hline
\end{tabular}

\section{Dicotyledones}

\begin{tabular}{|r|r|r|r|r|}
\hline & Aizoaceae & $\begin{array}{r}\text { Carpobrotus edulis (L.) N. E. } \\
\text { Brown in Philip. }\end{array}$ & Geo & Plu. \\
\hline 81 & & Amaranthaceae & Amaranthus blithoides S. \\
Watson. & Th & Med./ Eur-Si. \\
\hline 82 & Amaranthaceae & Amaranthus retroflexus L. & Th & Med./ Eur-Si \\
\hline 83 & $"$ & Amaranthus viridis L. & Th & Trop. \\
\hline 84 & Anacardiaceae & Pistacia lentiscus L. & NP & Med./ Ir-Tu. \\
\hline 85 & $"$ & Rhus tripartita (Ucria.) Grande. & NP & Med. \\
\hline 86 & Apiaceae & Ammi majus L. & Th & Med. \\
\hline 87 & $"$ & Anethum graveolens L. & Th & Med./ Ir-Tu. \\
\hline 88 & $"$ & Bunium fontainesii (Pers.) Maire. & Geo & Med. \\
\hline 89 & $"$ & Bupleurum lancifolium Hornem. & Th & Med./ Ir-Tu. \\
\hline 90 & $"$ & Bupleurum gibraltaricum Lam. & Ch & Plu. \\
\hline 91 & $"$ & Bupleurum odontites L. & Th & Med. \\
\hline 92 & $"$ & Bupleurum semicopositum L. & Th & Med./ Ir-Tu. \\
\hline 93 & $"$ & Bupleurum trichopodum Boiss. & Th & Med. \\
\hline
\end{tabular}




\begin{tabular}{|c|c|c|c|c|}
\hline 94 & $"$ & Daucus capillifolius Gilli. & Th & Med. \\
\hline 95 & " & Daucus jordanicus Bost. & $\mathrm{Th}$ & Med./ Sah-Ar. \\
\hline 96 & $"$ & Dancus syrticus Murb. & Th & Med. \\
\hline 97 & $"$ & Ferula tingitana $\mathrm{L}$. & $\mathrm{H}$ & Med. \\
\hline 98 & $"$ & Pimpinella peregrina $\mathrm{L}$. & $\mathrm{H}$ & Med. \\
\hline 99 & " & $\begin{array}{r}\text { Pituranthos tortuousus (Desf.) } \\
\text { Benth \& Hok. }\end{array}$ & $\mathrm{Ch}$ & Med. \\
\hline 100 & $"$ & Scandix australis L. & Th & Med. \\
\hline 101 & $"$ & Scandix pectin-veneris $\mathrm{L}$ & Th & Med./ Eur-Si. \\
\hline 102 & $"$ & Torilis leptophylla L. & $\mathrm{Th}$ & Med./ Ir-Tu. \\
\hline 103 & " & Torilis nodosa (L.) Gaertn. & $\mathrm{Th}$ & $\begin{array}{l}\text { Med./ Ir-Tu./ } \\
\text { Eur-Si. }\end{array}$ \\
\hline 104 & $"$ & Torilis tenella Del. & Th & Med. \\
\hline 105 & Asclepiacaceae & $\begin{array}{r}\text { Caralluma europaea (Guss.) } \\
\text { N.E.Br. }\end{array}$ & $\mathrm{H}$ & Med. \\
\hline 106 & $"$ & Calotropis procera (Ait.) Ait. & NP & Sud./ Sah-Ar. \\
\hline 107 & $"$ & Periploca angustifolia Labill . & $\mathrm{NP}$ & Med. \\
\hline 108 & Asteraceae & Amberboa libyca (Viv.)Alavi & $\mathrm{Th}$ & Med. \\
\hline 109 & $"$ & Amberboa lippii ( L ) DC. & $\mathrm{Th}$ & Sah-Ar. \\
\hline 110 & $"$ & Amberboa tubiflora Murb. & Th & Med. \\
\hline 111 & $"$ & Anacyclus clavatus (Desf.) Pers. & Th & Med. \\
\hline 112 & $"$ & Anacyclus monanthos (L.) Thell. & $\mathrm{Th}$ & Med. \\
\hline 113 & $"$ & Andryala integrifolia $\mathrm{L}$. & Th & Med. \\
\hline 114 & $"$ & Anthemis secundiramea Biv. & $\mathrm{Th}$ & Med. \\
\hline 115 & $"$ & Artemisia campestris L. & $\mathrm{H}$ & Med./ Eur-Si. \\
\hline 116 & $"$ & Asteriscus pygmaeus DC. & $\mathrm{Th}$ & Ir-Tu./ Sah-Ar. \\
\hline 117 & " & $\begin{array}{r}\text { Atractylis cadruus (Forsk.) } \\
\text { Christ in Dansk. }\end{array}$ & $\mathrm{H}$ & Sah-Ar. \\
\hline 118 & $"$ & Atractylis cancellata $\mathrm{L}$. & $\mathrm{Th}$ & Med. \\
\hline 119 & $"$ & Atractylis serrata Pomel & $\mathrm{Th}$ & Med. \\
\hline 120 & $"$ & $\begin{array}{r}\text { Atractylis serratuloides Sieb. ex } \\
\text { Cass. }\end{array}$ & $\mathrm{H}$ & Sah-Ar. \\
\hline 121 & $"$ & Bombycilaena discolorPers. & $\mathrm{Th}$ & Med. \\
\hline 122 & $"$ & Calendula arvensis $\mathrm{L}$. & $\mathrm{Th}$ & Med./ Ir-Tu. \\
\hline 123 & " & $\begin{array}{r}\text { Carduncellus pinnatus (Desf.) } \\
\text { DC. }\end{array}$ & $\mathrm{H}$ & Med. \\
\hline 124 & " & $\begin{array}{r}\text { Carduus argentatus Durieu in } \\
\text { Duchartre. }\end{array}$ & $\mathrm{Th}$ & Med. \\
\hline 125 & $"$ & Carduns getulus Pomel & $\mathrm{Th}$ & Sah-Ar \\
\hline 126 & $"$ & Carlina involucrata Boint. & $\mathrm{Th}$ & Med. \\
\hline 127 & $"$ & Carlina sicula Ten. & $\mathrm{Th}$ & Med. \\
\hline 128 & " & Carthamus lanatus L. & $\mathrm{Th}$ & $\begin{array}{l}\text { Med./ Ir-Tu./ } \\
\text { Eur-Si. }\end{array}$ \\
\hline 129 & $"$ & Centaurea africana Lam. & $\mathrm{H}$ & Med. \\
\hline 130 & $"$ & Centaurea alexandrina Delile & $\mathrm{Th}$ & Med. \\
\hline 131 & $"$ & Centaurea dimorpha Viv. & $\mathrm{H}$ & Med./ Ir-Tu. \\
\hline 132 & $"$ & Centaurea glomerata Vahl. & $\mathrm{Th}$ & Med. \\
\hline 133 & $"$ & Centaurea maroccana Ball. & $\mathrm{Th}$ & Med. \\
\hline 134 & $"$ & Centaurea melitensis $\mathrm{L}$. & $\mathrm{Th}$ & Med./ Eur-Si. \\
\hline
\end{tabular}




\begin{tabular}{|c|c|c|c|c|}
\hline 135 & $"$ & Centaurea sphaerocephala L. & $\mathrm{H}$ & Med. \\
\hline 136 & $"$ & Chamomilla aurea Loefl & $\mathrm{Th}$ & Med./ Ir-Tu. \\
\hline 137 & $"$ & $\begin{array}{r}\text { Chrysanthemum carinatum } \\
\text { Schousboe }\end{array}$ & $\mathrm{Th}$ & Med./ Eur-Si \\
\hline 138 & $"$ & Chrysanthemum coronarium L. & Th & Med. \\
\hline 139 & $"$ & Cichorium pumilum Jacq & Th & Med./ Ir-Tu. \\
\hline 140 & $"$ & Conyza aegyptiaca (L.)Dryander & Th & Med. \\
\hline 141 & $"$ & Conyza bonariensis $\mathrm{L}$ & Th & Med. \\
\hline 142 & $"$ & Conyza canadensis $\mathrm{L}$ & $\mathrm{Th}$ & Cos. \\
\hline 143 & $"$ & Crepis libyca Pamp. & $\mathrm{H}$ & Med. \\
\hline 144 & $"$ & Crepis senecioides Delile. & Th & Med. \\
\hline 145 & $"$ & Crepis vesicaria $\mathrm{L}$. & $\mathrm{H}$ & Med./ Eur-Si. \\
\hline 146 & " & $\begin{array}{r}\text { Crupina crupinastrum (Moris) } \\
\text { Vis. }\end{array}$ & Th & Med./ Ir-Tu. \\
\hline 147 & $"$ & Cynara cardunculus L. & $\mathrm{H}$ & Med. \\
\hline 148 & $"$ & Echinops galalensis Schweinf. & $\mathrm{H}$ & Med. \\
\hline 149 & " & Echinops hirsutissimum Turra. & $\mathrm{H}$ & Med. \\
\hline 150 & $"$ & Filago desertorum Pomel & Th & Ir-Tu./ Sah-Ar. \\
\hline 151 & $"$ & Filago pyramidata L. & $\mathrm{Th}$ & Med./ Ir-Tu. \\
\hline 152 & $"$ & $\begin{array}{r}\text { Hedypnois cretica (L.) Dum.- } \\
\text { Courset }\end{array}$ & Th & Med. \\
\hline 153 & $"$ & Helichrysum stoechas (L.)Moench & $\mathrm{H}$ & Med. \\
\hline 154 & $"$ & Hyoseris scabra L. & Th & Med. \\
\hline 155 & $"$ & Hypochoeris achyrophorus L. & $\mathrm{Th}$ & Med. \\
\hline 156 & $"$ & Hypochoeris glabra L. & Th & Med. \\
\hline 157 & " & Koelpinia linearis Pallas. & Th & Med./ Eur-Si. \\
\hline 158 & " & Launaea nudicaulis $\mathrm{L}$. & $\mathrm{H}$ & $\begin{array}{r}\text { Sah-Ar./ Sud. /Ir- } \\
\text { Tu. }\end{array}$ \\
\hline 159 & $"$ & Launaea procumbens Roxb. & $\mathrm{H}$ & Med./ Ir-Tu. \\
\hline 160 & " & $\begin{array}{r}\text { Lannaea resedifolia (L.) O. } \\
\text { Kuntze }\end{array}$ & $\mathrm{H}$ & Med. \\
\hline 161 & $"$ & Leontodon hispidulus Delile. & Th & Med./ Ir-Tu. \\
\hline 162 & $"$ & Leontodon simplex (Viv.) Widder & Th & Med./ Eur-Si. \\
\hline 163 & $"$ & Leontodon tuberosus $\mathrm{L}$ & $\mathrm{H}$ & Med. \\
\hline 164 & $"$ & Nolletia chrysocomides Desf. & $\mathrm{H}$ & Med. \\
\hline 165 & $"$ & Notobasis syriaca (L.) Cass. & Th & Med./ Ir-Tu. \\
\hline 166 & $"$ & Onopordum confusum Pamp. & $\mathrm{H}$ & Med. \\
\hline 167 & " & $\begin{array}{r}\text { Onopordum espinae Cosson } \\
\text { exBonnet }\end{array}$ & $\mathrm{H}$ & Med. \\
\hline 168 & $"$ & Pallenis spinosa (L.) Cass. & $\mathrm{H}$ & Med./ Ir-Tu. \\
\hline 169 & $"$ & Phagnalon rupestre (L.) DC. & $\mathrm{H}$ & Med./ Ir-Tu. \\
\hline 170 & $"$ & Picris asplenoides $\mathrm{L}$. & $\mathrm{Th}$ & Sah-Ar. \\
\hline 171 & $"$ & Reichardia tingitana (L.)Roth & Th & Ir-Tu./ Sah-Ar. \\
\hline 172 & " & $\begin{array}{r}\text { Rhagadiolus stellatus } \\
\text { (L.)Gaertner }\end{array}$ & $\mathrm{Th}$ & Med./ Ir-Tu. \\
\hline 173 & $"$ & Scorzonera undulata Vahl & Geo & Med. \\
\hline 174 & $"$ & Senecio gallicus Chiax & $\mathrm{Th}$ & Med. \\
\hline 175 & " & Silybum marianum (L.) Gaertner & Th & $\begin{array}{l}\text { Med./ Ir-Tu./ } \\
\text { Eur-Si }\end{array}$ \\
\hline 176 & $"$ & Sonchus asper (L.)Hill & $\mathrm{H}$ & Med./ Ir-Tu. \\
\hline
\end{tabular}




\begin{tabular}{|c|c|c|c|c|}
\hline 177 & $"$ & Sonchus oleraceus L. & Th & Cos. \\
\hline 178 & $"$ & Sonchus tenerrimus L. & $\mathrm{Th}$ & Med./ Ir-Tu./ Sud. \\
\hline 179 & " & $\begin{array}{r}\text { Tripleurospermum trifuscatum } \\
\text { (Desf.) Schultz }\end{array}$ & $\mathrm{Th}$ & Med. \\
\hline 180 & $"$ & Urospermum delachampii $\mathrm{L}$. & $\mathrm{H}$ & Med. \\
\hline 181 & " & $\begin{array}{r}\text { Urospermum picroides (L.) Scop. } \\
\text { Ex Schmidt. }\end{array}$ & Th & Med./ Ir-Tu. \\
\hline 182 & $"$ & $\begin{array}{r}\text { Verbasina encelioides (Cav.) } \\
\text { Benth. \& Hook. }\end{array}$ & Th & Americas \\
\hline 183 & $"$ & Xanthium spinosum L & $\mathrm{Th}$ & Boreal-Trop. \\
\hline 184 & Boraginaceae & Alkanna tinctoria (L.) Tausch. & $\mathrm{H}$ & Med. \\
\hline 185 & " & Arnebia decumbens Vent. & Th & Med./ Ir-Tu. \\
\hline 186 & " & $\begin{array}{r}\text { Buglossoides tenuiflora (L.f.) I.M. } \\
\text { Johnst. }\end{array}$ & Th & Med./ Ir-Tu. \\
\hline 187 & $"$ & Cynoglossum cheirifolium $\mathrm{L}$. & Th & Med. \\
\hline 188 & " & Echiochilon fruticosum Desf. & $\mathrm{Ch}$ & Med. \\
\hline 189 & $\bar{~}$ & Echium angustifolium Mill. & $\mathrm{H}$ & Med. \\
\hline 190 & $"$ & Echium bumile Desf. & $\mathrm{H}$ & Med. \\
\hline 191 & " & Elizaldia calycina Roem. & $\mathrm{Th}$ & Med. \\
\hline 192 & $"$ & Heliotropium europaeum L. & $\mathrm{Th}$ & Med. \\
\hline 193 & $"$ & Lappula spinocarpos Forsk. & Th & Med./ Ir-Tu. \\
\hline 194 & " & $\begin{array}{r}\text { Neatostema apulum (L.) I.M. } \\
\text { Johnst. }\end{array}$ & Th & Med. \\
\hline 195 & " & $\begin{array}{r}\text { Nonea micrantha Boiss. \& } \\
\text { Reuter }\end{array}$ & Th & Med. \\
\hline 196 & Brassicaceae & Biscutella didyma $\mathrm{L}$. & $\mathrm{Th}$ & Med./ Ir-Tu. \\
\hline 197 & $"$ & Brassica tournefortii Gouan. & Th & Med./ Sah-Ar. \\
\hline 198 & $"$ & Cakile aegyptiaca (L.) Willd. & Th & Med./ Eur-Si. \\
\hline 199 & " & $\begin{array}{r}\text { Capsella bursa-pastoris (L.) } \\
\text { Medik. }\end{array}$ & Th & Plu. \\
\hline 200 & $"$ & Cardaria draba L .Desv. & Th & Med./ Eur-Si. \\
\hline 201 & " & Carrichtera annua (L.) DC. & Th & $\begin{array}{l}\text { Med./ Ir-Tu./ } \\
\text { Eur-Si. }\end{array}$ \\
\hline 202 & " & Clypeola jonthlaspi L. & $\mathrm{Th}$ & $\begin{array}{r}\text { Med./ Ir-Tu./ } \\
\text { Eur-Si. }\end{array}$ \\
\hline 203 & $"$ & Didesmus aegyptius L \& Desv. & Th & Med. \\
\hline 204 & $"$ & Didesmus bipinnatus (Desf.) DC. & Th & Med. \\
\hline 205 & $"$ & Diplotaxis harra (Forsk.)Boiss. & Th & Med./ Ir-Tu. \\
\hline 206 & $"$ & Diplotaxis muralis (L.) DC. & Th & Med./ Eur-Si. \\
\hline 207 & " & $\begin{array}{r}\text { Enarthrocarpus clavatus Del. ex } \\
\text { Godr. }\end{array}$ & $\mathrm{Th}$ & Med. \\
\hline 208 & $"$ & Eruca longirostris Uechtr. & Th & Med. \\
\hline 209 & $"$ & Eruca sativa Mill. & Th & Med./ Ir-Tu. \\
\hline 210 & $"$ & Eurcaria microcarpa Boiss. & Th & Med./ Sah-Ar. \\
\hline 211 & $"$ & Lepidium sativum L & $\mathrm{Th}$ & Plu. \\
\hline 212 & $"$ & Lobularia libyca (Viv.) Meisner. & Th & Med./ Ir-Tu. \\
\hline 213 & $"$ & Lobularia maritima L \& Desv. & $\mathrm{H}$ & Med. \\
\hline 214 & $"$ & Lonchophora kralikii Pomel & Th & Med. \\
\hline 215 & $"$ & Matthiola longipetala (Vent.) DC. & Th & Med./ Ir-Tu. \\
\hline 216 & $"$ & Matthiola parviflora (Schousbe.) & Th & Sah-Ar. \\
\hline
\end{tabular}




\begin{tabular}{|c|c|c|c|c|}
\hline & & R.Br. In Ait. & & \\
\hline 217 & $"$ & Rapistrum rugosum (L.) All. & Th & Med./ Ir-Tu. \\
\hline 218 & $"$ & Sinapis alba $\mathrm{L}$. & Th & $\begin{array}{r}\text { Med./ Ir-Tu./ } \\
\text { Eur-Si. }\end{array}$ \\
\hline 219 & $"$ & Sinapisflexuosa Poir. & Th & Med. \\
\hline 220 & $"$ & Sinapis pubescens L. & $\mathrm{Th}$ & Med. \\
\hline 221 & $"$ & Sisymbrium erysimoides Desf. & $\mathrm{Th}$ & Med./ Ir-Tu. \\
\hline 222 & $"$ & Sisymbrium irio $\mathrm{L}$ & Th & Med./ Ir-Tu. \\
\hline 223 & Cactaceae & Opuntia ficus-indica (L.) Mill. & NP & Med./ Trop. \\
\hline 224 & Caeslpiniaceae & Ceratonia siliqua L. & $\mathrm{Ph}$ & Med. \\
\hline 225 & Capparaceae & Capparis spinosa $\mathrm{L}$ & $\mathrm{NP}$ & Med. \\
\hline 226 & Caryophyllaceae & Arenaria serpyllifolia L. & Th & $\begin{array}{r}\text { Med./ Ir-Tu./ } \\
\text { Eur-Si. }\end{array}$ \\
\hline 227 & $\bar{"}$ & Cerastium glomeratum Thuill. & Th & $\begin{array}{r}\text { Med./ Ir-Tu./ } \\
\text { Eur-Si. }\end{array}$ \\
\hline 228 & $"$ & Cerastium pumilum Curtis. & Th & Med./ Ir-Tu. \\
\hline 229 & $"$ & Minuartia bybrida Vill. & Th & Med./ Ir-Tu. \\
\hline 230 & $"$ & Polycarpon tetraphyllum L. & $\mathrm{Th}$ & Med./ Eur-Si. \\
\hline 231 & $"$ & Silene apetala Willd. & Th & Med./ Ir-Tu. \\
\hline 232 & " & Silene behen $\mathrm{L}$. & Th & Med. \\
\hline 233 & $"$ & Silene cerastioides L. & $\mathrm{Th}$ & Med. \\
\hline 234 & $"$ & Silene colorata Poiret. & Th & Med. \\
\hline 235 & $"$ & Silene gallica $\mathrm{L}$ & Th & Cos. \\
\hline 236 & " & Silene tridentata Desf. & Th & Med. \\
\hline 237 & $"$ & Silene villosa Forsk. & Th & Med. \\
\hline 238 & $"$ & Silene viviani Teud. & Th & Med. \\
\hline 239 & " & $\begin{array}{r}\text { Spergularia bocconii (Sol.) Ash et } \\
\text { Grbn. }\end{array}$ & Th & Med./ Ir-Tu. \\
\hline 240 & " & $\begin{array}{r}\text { Spergularia diandra (Guss.) } \\
\text { Heldr. \& Sart. }\end{array}$ & Th & $\begin{array}{l}\text { Med./ Ir-Tu./ } \\
\text { Eur-Si. }\end{array}$ \\
\hline 241 & Chenopodiaceae & Beta vulgaris L. & Th & $\begin{array}{r}\text { Med./ Ir-Tu./ } \\
\text { Eur-Si. }\end{array}$ \\
\hline 242 & " & Chenopodium album L. & Th & Plu. \\
\hline 243 & $"$ & Chenopodium murale L. & Th & Plu. \\
\hline 244 & $"$ & Salsola kali L. & $\mathrm{Th}$ & Plu. \\
\hline 245 & eae & Cistus parviflorus Lam. & $\mathrm{Ch}$ & Med. \\
\hline 246 & " & Cistus salvifolius L. & $\mathrm{Ch}$ & Med. \\
\hline 247 & $"$ & Fumana arabica (L.) Spach. & $\mathrm{Ch}$ & Med. \\
\hline 248 & $"$ & Fumana laevipes (L.) Spach. & $\mathrm{Ch}$ & Med. \\
\hline 249 & " & $\begin{array}{r}\text { Fumana themifolia (L.) Spach ex } \\
\text { Webb. }\end{array}$ & $\mathrm{Ch}$ & Med. \\
\hline 250 & $"$ & Helianthemum hirtum L & $\mathrm{Ch}$ & Med. \\
\hline 251 & " & Helianthemum kahiricum Delile. & $\mathrm{Ch}$ & Med. \\
\hline 252 & $"$ & Helianthemum ledifolium L. Mill & Th & Med. \\
\hline 253 & $\bar{~}$ & Helianthemum lippii (L). Dum. & $\mathrm{Ch}$ & Med. \\
\hline 254 & 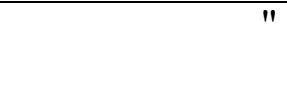 & $\begin{array}{r}\text { Helianthemum salicifolium (L.) } \\
\text { Mille,r }\end{array}$ & Th & $\begin{array}{l}\text { Med./ Ir-Tu./ } \\
\text { Eur-Si. }\end{array}$ \\
\hline 255 & $"$ & Helianthemum stipulatum Forsk. & $\mathrm{Ch}$ & Med. \\
\hline 256 & 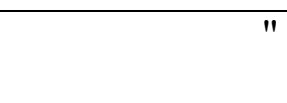 & $\begin{array}{r}\text { Helianthemum virgatum (Desf). } \\
\text { Pers. }\end{array}$ & $\mathrm{Ch}$ & Med. \\
\hline
\end{tabular}




\begin{tabular}{|c|c|c|c|c|}
\hline 257 & $"$ & Tuberaria guttata (L.) Fourr. & $\mathrm{Th}$ & Med./ Eur-Si. \\
\hline 258 & Convolvulaceae & Convolvulus altheoides L. & $\mathrm{Th}$ & Med. \\
\hline 259 & $"$ & Convolvulus arvensis $\mathrm{L}$. & Geo & Plu. \\
\hline 260 & $"$ & Convolvulus dorycnium L. & $\mathrm{H}$ & Med. \\
\hline 261 & " & $\begin{array}{r}\text { Convolvulus oleifolius Desr. in } \\
\text { Lam. }\end{array}$ & $\mathrm{Ch}$ & Med. \\
\hline 262 & $"$ & Convolvulus siculus L. & $\mathrm{Th}$ & Med. \\
\hline 263 & $"$ & Convolvulus supinus Coss. & $\mathrm{Th}$ & Med. \\
\hline 264 & Coridaceae & Coris monspeliensis L. & $\mathrm{Th}$ & Med. \\
\hline 265 & Crassulaceae & Crassula alata (Viv) Berg. & $\mathrm{Th}$ & Med./ Ir-Tu. \\
\hline 266 & $"$ & Sedum album L & $\mathrm{Th}$ & Med./ Ir-Tu. \\
\hline 267 & $"$ & Sedum sediforme (Jacq.) Pau & $\mathrm{H}$ & Med. \\
\hline 268 & " & $\begin{array}{r}\text { Umbilicus horizontalis (Guss.) } \\
\text { DC. }\end{array}$ & $\mathrm{H}$ & Med. \\
\hline 269 & " & Umbilicus rupestris Salisb & $\mathrm{H}$ & Med. \\
\hline 270 & Cucurbitaceae & Bryonia cretica $\mathrm{L}$. & $\mathrm{H}$ & Med./ Ir-Tu. \\
\hline 271 & " & Citrullus colocynthis (L.) Schrad. & $\mathrm{H}$ & Sah-Ar. \\
\hline 272 & Cuscutaceae & Cuscuta planiflora Ten. & $\mathrm{Th}$ & Med./ Ir-Tu. \\
\hline 273 & Dipsacaceae & Scabiosa arenaria Forsk. & $\mathrm{Th}$ & Med. \\
\hline 274 & " & Scabiosa monspeliensis Jacq. & $\mathrm{Th}$ & Med. \\
\hline 275 & Euphorbiaceae & $\begin{array}{r}\text { Chrozophora obliqua (Vahl.) Juss. } \\
\text { Ex Spreng }\end{array}$ & $\mathrm{Th}$ & Med./ Ir-Tu. \\
\hline 276 & $"$ & Crozophora tinctoria (L.) Juss. & $\mathrm{Th}$ & Med./ Ir-Tu. \\
\hline 277 & " & Euphorbia bivonae Steud. & $\mathrm{Ch}$ & Med. \\
\hline 278 & $"$ & Euphorbia exigua L. & $\mathrm{Th}$ & Med./ Ir-Tu. \\
\hline 279 & $"$ & Euphorbia falcata L. & $\mathrm{Th}$ & Trop. \\
\hline 280 & " & Euphorbia helioscopia $\mathrm{L}$. & $\mathrm{Th}$ & Plu. \\
\hline 281 & $"$ & Euphorbia parvula Delile. & $\mathrm{Th}$ & Med. \\
\hline 282 & $"$ & Euphorbia peplus L. & $\mathrm{Th}$ & Sud. \\
\hline 283 & " & Euphorbia terracina $\mathrm{L}$ & $\mathrm{H}$ & Med./ Eur-Si. \\
\hline 284 & " & Mercurialis annua $\mathrm{L}$. & $\mathrm{Th}$ & Med. \\
\hline 285 & $"$ & Ricinus communis $\mathrm{L}$. & NP & Sud. \\
\hline 286 & Fabaceae & Anagyris foetida $\mathrm{L}$ & Th & Med. \\
\hline 287 & $"$ & Anthyllis tetraphylla $\mathrm{L}$. & $\mathrm{Th}$ & Med. \\
\hline 288 & " & Anthyllis vulneraria $\mathrm{L}$. & $\mathrm{Th}$ & Med. \\
\hline 289 & " & $\begin{array}{r}\text { Argyrolobium uniflorum (Decne.) } \\
\text { Jaub. \& Spach }\end{array}$ & $\mathrm{Ch}$ & Med. \\
\hline 290 & " & $\begin{array}{r}\text { Astragalus asterias Stev. ex } \\
\text { Ledeb. }\end{array}$ & $\mathrm{Th}$ & Med./ Ir-Tu. \\
\hline 291 & $"$ & Astragalus caprinus $\mathrm{L}$ & $\mathrm{H}$ & Med./ Ir-Tu. \\
\hline 292 & " & Astragalus hamosus $\mathrm{L}$. & Th & Med. \\
\hline 293 & " & Astragalus sinaicus Boiss & $\mathrm{Th}$ & Med./ Ir-Tu. \\
\hline 294 & $"$ & Astragalus stella Gouan. & $\mathrm{Th}$ & Med. \\
\hline 295 & $"$ & Astragalus tribuloides Del. & $\mathrm{Th}$ & Med./ Ir-Tu. \\
\hline 296 & " & Calicotome villosa (Poir.) Link. & NP & Med. \\
\hline 297 & $"$ & Coronilla repanda (Poir.) Guss & $\mathrm{Th}$ & Med. \\
\hline 298 & $"$ & Coronilla scorpioides L. \& Koch. & $\mathrm{Th}$ & Med. \\
\hline 299 & $"$ & Ebenus pinnata Ait. \& Hort. & $\mathrm{H}$ & Med. \\
\hline 300 & $"$ & Genista acanthocalda DC. & $\mathrm{NP}$ & Med. \\
\hline 301 & $"$ & Genista microcephala Coss. \& & $\mathrm{NP}$ & Med. \\
\hline
\end{tabular}




\begin{tabular}{|c|c|c|c|c|}
\hline & & Dur. & & \\
\hline 302 & $"$ & Hedysarum spinosissimum L. & $\mathrm{Th}$ & Med. \\
\hline 303 & " & Hippocrepis bicontorta Lois. & $\mathrm{Th}$ & Sah-Ar. \\
\hline 304 & $"$ & Hippocrepis ciliata Willd & $\mathrm{Th}$ & Med. \\
\hline 305 & $"$ & Hippocrepis multisiliquosa $\mathrm{L}$. & $\mathrm{Th}$ & Med. \\
\hline 306 & $"$ & Hippocrepis scabra DC & $\mathrm{H}$ & Med. \\
\hline 307 & $"$ & Hymenocarpos circinatus (L.) Savi. & $\mathrm{Th}$ & Med./ Ir-Tu. \\
\hline 308 & $"$ & Lathyrus cicera $\mathrm{L}$. & $\mathrm{Th}$ & Med./ Ir-Tu. \\
\hline 309 & $"$ & Lotus cytisoides $\mathrm{L}$. & $\mathrm{H}$ & Med. \\
\hline 310 & $"$ & Lotus edulis L. & $\mathrm{Th}$ & Med. \\
\hline 311 & $"$ & Lotus halophilus Boiss. & $\mathrm{Th}$ & Med. \\
\hline 312 & $"$ & Lotus ornithopodioides L. & $\mathrm{Th}$ & Med. \\
\hline 313 & $"$ & Medicago coronata (L.) Bart. & Th & Med. \\
\hline 314 & $"$ & Medicago laciniata $\mathrm{L}$. & Th & Sah-Ar. \\
\hline 315 & " & $\begin{array}{r}\text { Medicago littoralis Rohde. ex } \\
\text { Lois. }\end{array}$ & $\mathrm{Th}$ & Med. \\
\hline 316 & $"$ & Medicago minima (L.) Bart. & $\mathrm{Th}$ & Med./ Ir-Tu. \\
\hline 317 & $"$ & Medicago polymorpha $\mathrm{L}$. & $\mathrm{Th}$ & Med./ Ir-Tu. \\
\hline 318 & $"$ & Medicago sativa $\mathrm{L}$. & $\mathrm{H}$ & Med. \\
\hline 319 & " & Medicago tornata (L.) Mill. & $\mathrm{Th}$ & Med. \\
\hline 320 & $"$ & Melilotus indicus (L.) All. & $\mathrm{Th}$ & Med. \\
\hline 321 & $"$ & Melilotus sulcatus Desf. & $\mathrm{Th}$ & Med. \\
\hline 322 & $"$ & Ononis natrix $\mathrm{L}$. & $\mathrm{Ch}$ & Med. \\
\hline 323 & $"$ & Ononis ornithopodioides L. & $\mathrm{Th}$ & Med. \\
\hline 324 & $"$ & Ononis reclinata $\mathrm{L}$ & $\mathrm{Th}$ & Med./ Ir-Tu. \\
\hline 325 & $"$ & Ononis serrata Forsk. & $\mathrm{Th}$ & Med./ Ir-Tu. \\
\hline 326 & $"$ & Ononis sicula Guss. & Th & Med./ Ir-Tu. \\
\hline 327 & $"$ & Ononis viscosa $\mathrm{L}$. & $\mathrm{Th}$ & Med. \\
\hline 328 & $"$ & Psoralea bituminosa $\mathrm{L}$. & $\mathrm{H}$ & Med. \\
\hline 329 & $"$ & Retama raetam (Forsk.) Webb & $\mathrm{NP}$ & Sah-Ar. \\
\hline 330 & $"$ & Scorpiurs muricatus L. & $\mathrm{Th}$ & Med. \\
\hline 331 & $"$ & Scorpiurs subbvillosus (L.) Lam & $\mathrm{Th}$ & Med. \\
\hline 332 & " & $\begin{array}{r}\text { Spartidium saharae (Coss. et } \\
\text { Dur.) Pomel. }\end{array}$ & $\mathrm{NP}$ & Sah-Ar. \\
\hline 333 & $"$ & Tetragonolobus purpureus Moench & $\mathrm{Th}$ & Med. \\
\hline 334 & $"$ & Trifolium campestre Schreb. & $\mathrm{Th}$ & Med. \\
\hline 335 & $"$ & Trifolium cherleri $\mathrm{L}$. & $\mathrm{Th}$ & Med. \\
\hline 336 & $"$ & Trifolium scabrum L. & $\mathrm{Th}$ & Med. \\
\hline 337 & $"$ & Trifolium stellatum $\mathrm{L}$. & Th & Med. \\
\hline 338 & " & Trifolium tomentosum $\mathrm{L}$. & $\mathrm{Th}$ & $\begin{array}{r}\text { Med./ Ir-Tu./ } \\
\text { Eur-Si. }\end{array}$ \\
\hline 339 & $"$ & Trigonella stellata Forsk. & Th & Med./ Ir-Tu. \\
\hline 340 & $" 1$ & Vicia laxiflora Brot. & $\mathrm{Th}$ & Med. \\
\hline 341 & " & Vicia lutea $\mathrm{L}$. & Th & Med. \\
\hline 342 & $"$ & Vicia monantha Retz. & $\mathrm{Th}$ & Med. \\
\hline 343 & " & Vicia sativa $\mathrm{L}$. & $\mathrm{Th}$ & $\begin{array}{r}\text { Med./ Ir-Tu./ } \\
\text { Eur-Si.. }\end{array}$ \\
\hline 344 & " & Vicia villosa Roth. & $\mathrm{Th}$ & $\begin{array}{r}\text { Med./ Ir-Tu./ } \\
\text { Eur-Si. }\end{array}$ \\
\hline 345 & Fumariaceae & Fumaria gaillardotii Boiss & $\mathrm{Th}$ & Med. \\
\hline
\end{tabular}




\begin{tabular}{|c|c|c|c|c|}
\hline 346 & $"$ & Fumaria parviflora Lam. & Th & Med./ Eur-Si. \\
\hline 347 & " & Fumaria vaillantii Lois. & Th & Plu. \\
\hline 348 & Gentianaceae & $\begin{array}{r}\text { Centaurium pulchellum (Swartz.) } \\
\text { Druce. }\end{array}$ & Th & Med. \\
\hline 349 & Geraniaceae & Erodium arborescens Desf. & $\mathrm{H}$ & Sah-Ar. \\
\hline 350 & $"$ & Erodium cicutarium $\mathrm{L}$ & Th & Med. \\
\hline 351 & $"$ & $\begin{array}{r}\text { Erodium glaucophyllum (L.) L' } \\
\text { Herit. }\end{array}$ & $\mathrm{H}$ & Sah-Ar. \\
\hline 352 & $"$ & Erodium birtum (Frorsk.) Will. & Th & Sah-Ar. \\
\hline 353 & $"$ & Erodium laciniatum (Cav.) Willd. & Th & Med. \\
\hline 354 & $"$ & Erodium malacoides (L.) L Her. & Th & Med./ Ir-Tu. \\
\hline 355 & $"$ & Erodium moschatum (L.) L Her. & $\mathrm{Th}$ & Med. \\
\hline 356 & " & Geranium molle L. & Th & Med./ Eur-Si \\
\hline 357 & Globulariaceae & Globularia alypum L. & $\mathrm{Ch}$ & Med. \\
\hline 358 & Hypecoaceae & Hypecoum geslini Coss. et Kral. & $\mathrm{Th}$ & Med. \\
\hline 359 & $"$ & Hypecoum procumbens L. & $\mathrm{Th}$ & Med. \\
\hline 361 & Illcebraceae & Gymnocarpos decander Forsk. & $\mathrm{Ch}$ & Med./ Ir-Tu. \\
\hline 360 & $"$ & Herniaria cinerea DC. & $\mathrm{Th}$ & Med./ Ir-Tu. \\
\hline 362 & " & $\begin{array}{r}\text { Herniaria fontanesii J.Gay in } \\
\text { Duch. }\end{array}$ & $\mathrm{H}$ & Med. \\
\hline 363 & " & $\begin{array}{r}\text { Herniaria hemistemon J.Gay in } \\
\text { Duch. }\end{array}$ & $\mathrm{H}$ & Med./ Ir-Tu. \\
\hline 364 & $"$ & Paronychia arabica (L.) DC. & $\mathrm{Th}$ & Med./ Ir-Tu. \\
\hline 365 & " & Paronychia capitata (L.) Lamk. & $\mathrm{H}$ & Med. \\
\hline 366 & Lamiaceae & Ajuga iva (L.) Schreber & $\mathrm{H}$ & Med./ Ir-Tu. \\
\hline 367 & " & Lamium amplexicaule L. & Th & Med. \\
\hline 368 & " & Lavandula multifida $\mathrm{L}$. & $\mathrm{Ch}$ & Med./ Ir-Tu. \\
\hline 369 & $"$ & Marrubium alysson L. & $\mathrm{H}$ & Med. \\
\hline 370 & $"$ & Marrubium vulgare $\mathrm{L}$. & $\mathrm{H}$ & Med./ Ir-Tu. \\
\hline 371 & " & $\begin{array}{r}\text { Micromeria nervosa (Desf.) } \\
\text { Benth. }\end{array}$ & $\mathrm{Ch}$ & Med. \\
\hline 372 & " & Prasium majus L. & $\mathrm{NP}$ & Med. \\
\hline 373 & $"$ & Rosmarinus officinalis $\mathrm{L}$. & $\mathrm{Ch}$ & Med. \\
\hline 374 & $"$ & Salvia lanigera Poir. & Th & Med./ Ir-Tu. \\
\hline 375 & " & Salvia verbenaca $\mathrm{L}$. & Th & $\begin{array}{r}\text { Med./ Ir-Tu./ } \\
\text { Eur-Si. }\end{array}$ \\
\hline 376 & $"$ & Sideritis montana L. & Th & Med./ Ir-Tu. \\
\hline 377 & $"$ & Teucrium polium L. & $\mathrm{Ch}$ & $\begin{array}{l}\text { Med./ Ir-Tu./ } \\
\text { Eur-Si. }\end{array}$ \\
\hline 378 & $"$ & Thymus algeriensis Boiss & $\mathrm{Ch}$ & Med. \\
\hline 379 & $"$ & $\begin{array}{r}\text { Thymus capitatus (L.) Hoffm. \& } \\
\text { Link }\end{array}$ & $\mathrm{Ch}$ & Med. \\
\hline 380 & haceae & Linum bienne Mill. & Th & Med./ Ir-Tu. \\
\hline 381 & 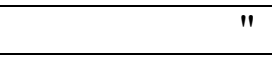 & Linum strictum $\mathrm{L}$. & Th & Med. \\
\hline 382 & " & Linum trigynum L. & Th & $\begin{array}{r}\text { Med./ Ir-Tu./ } \\
\text { Eur-Si. }\end{array}$ \\
\hline 383 & $"$ & Linum usitatissimum $\mathrm{L}$ & $\mathrm{Th}$ & Med. \\
\hline 384 & Malvaceae & Malva aegyptia $\mathrm{L}$ & $\mathrm{Th}$ & Sah-Ar. \\
\hline 385 & 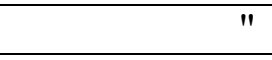 & Malva parviflora L. & Th & Med./ Eur-Si. \\
\hline 386 & " & Malva sylvestris $\mathrm{L}$. & $\mathrm{H}$ & Med./ Ir-Tu. \\
\hline
\end{tabular}




\begin{tabular}{|c|c|c|c|c|}
\hline 387 & Mimosaceae & Acacia cyanophylla Lindley. & $\mathrm{Ph}$ & Ir-Tu. \\
\hline 388 & $"$ & Acacia karroo Hayne & $\mathrm{Ph}$ & Plu. \\
\hline 389 & $"$ & Acacia nolitica (L.) Delile. & $\mathrm{Ph}$ & Plu. \\
\hline 390 & Moraceae & Ficus carica $\mathrm{L}$. & $\mathrm{Ph}$ & Med. \\
\hline 391 & Myrtaceae & Eucalyptus cosmophylla F. muell. & $\mathrm{Ph}$ & Australia. \\
\hline 392 & $"$ & Eucalyptus gomphocephala DC. & $\mathrm{Ph}$ & Australia. \\
\hline 393 & " & $\begin{array}{r}\text { Eucalyptus leucoxylon F. Muell. } \\
\text { In Trans. }\end{array}$ & $\mathrm{Ph}$ & Australia. \\
\hline 394 & Oleaceae & Olea europaea L. & $\mathrm{Ph}$ & Med. \\
\hline 395 & Oxaidaceae & Oxalis articulata $\mathrm{S}$ avigny & Geo & Plu. \\
\hline 396 & " & Oxalis pes-caprae L. & Geo & Plu. \\
\hline 397 & Papaveraceae & Papaver bybridum L & Th & Med. \\
\hline 398 & " & Papaver rhoeas L. & Th & Med./ Ir-Tu. \\
\hline 399 & Plantaginaceae & Plantago afra $\mathrm{L}$ & $\mathrm{Th}$ & Med./ Ir-Tu. \\
\hline 400 & $"$ & Plantago albicans L. & $\mathrm{H}$ & Med./ Ir-Tu. \\
\hline 401 & " & Plantago amplexicaulis Cav. & Th & Med./ Ir-Tu. \\
\hline 402 & " & Plantago arenaria Walds.t \& Kit. & $\mathrm{Th}$ & $\begin{array}{r}\text { Med./ Ir-Tu./ } \\
\text { Eur-Si. }\end{array}$ \\
\hline 403 & $"$ & Plantago coronopus $\mathrm{L}$ & Th & Med./ Ir-Tu. \\
\hline 404 & " & Plantago lagopus $\mathrm{L}$ & $\mathrm{Th}$ & $\begin{array}{r}\text { Med./ Ir-Tu./ } \\
\text { Eur-Si. }\end{array}$ \\
\hline 405 & " & Plantago lanceolata $\mathrm{L}$ & $\mathrm{H}$ & $\begin{array}{r}\text { Med./ Ir-Tu./ Sah- } \\
\text { Ar. }\end{array}$ \\
\hline 406 & $"$ & Plantago notata Lag & Th & Med./ Ir-Tu. \\
\hline 407 & $"$ & Plantago ovata Forskal & $\mathrm{H}$ & Med./ Ir-Tu. \\
\hline 408 & $"$ & Plantago phaeostoma Boiss. & Th & Med. \\
\hline 409 & Plumbaginaceae & Limonium echioidesL.Mill. & Th & Med. \\
\hline 410 & $"$ & Limonium thouinii Viv. & $\mathrm{Th}$ & Sah-Ar. \\
\hline 411 & Polygonaceae & Emex spinosus L & $\mathrm{Th}$ & Med./ Ir-Tu. \\
\hline 412 & " & Polygonum equisetiforme Sibth. & $\mathrm{Ch}$ & Plu. \\
\hline 413 & $"$ & Rumex bucephalophorus L. & $\mathrm{Th}$ & Med. \\
\hline 414 & $"$ & Rumex tingitanus L. & $\mathrm{Th}$ & Ir-Tu. \\
\hline 415 & " & Rumex vesicarius L. & Th & Sah-Ar. \\
\hline 416 & Portulaceae & Portulaca oleracea & Th & $\begin{array}{r}\text { Med./ Ir-Tu./ } \\
\text { Eur-Si. }\end{array}$ \\
\hline 417 & Primulaceae & Anagallis arvensis L. & $\mathrm{Th}$ & $\begin{array}{r}\text { Med./ Ir-Tu./ } \\
\text { Eur-Si. }\end{array}$ \\
\hline 418 & $"$ & Anagallis monelli $\mathrm{L}$. & Th & Med. \\
\hline 419 & " & $\begin{array}{r}\text { Asterolinon linum-stellatum L. } \\
\text { Duby in DC. }\end{array}$ & $\mathrm{Th}$ & Med. \\
\hline 420 & Ranunculaceae & Adonins dentata Delile. & $\mathrm{Th}$ & Med./ Ir-Tu. \\
\hline 421 & $"$ & Adonis aestivalis $\mathrm{L}$. & Th & Med./ Ir-Tu. \\
\hline 422 & $"$ & Adonis microcarpa $\mathrm{DC}$ & $\mathrm{Th}$ & Med./ Ir-Tu. \\
\hline 423 & " & $\begin{array}{r}\text { Delphinium halteratum Sibth. \& } \\
\text { Smith. }\end{array}$ & $\mathrm{Th}$ & Med. \\
\hline 424 & $"$ & Nigella arvensis $\mathrm{L}$. & $\mathrm{Th}$ & Med./ Ir-Tu. \\
\hline 425 & $"$ & Nigella damascena L. & Th & Med./ Ir-Tu. \\
\hline 426 & $"$ & Ranunculus asiaticus $\mathrm{L}$. & $\mathrm{Th}$ & Med. \\
\hline 427 & $"$ & Ranunculus bullatus L. & Th & Med. \\
\hline 428 & Resedaceae & Reseda alba L. & $\mathrm{Th}$ & Med./ Ir-Tu./ \\
\hline
\end{tabular}




\begin{tabular}{|c|c|c|c|c|}
\hline & & & & Eur-Si. \\
\hline 429 & Rhamnaceae & Rhamnus alaternus $\mathrm{L}$. & $\mathrm{NP}$ & Med. \\
\hline 430 & " & Ziqiphus lotus (L.) Lam. & $\mathrm{NP}$ & Med./ Sud. \\
\hline 431 & Rosaceae & Sanguisorba minor Scop. & $\mathrm{Th}$ & Med. \\
\hline 432 & Rubiaceae & Callipeltis cucullaris L. & Th & Med./ Ir-Tu. \\
\hline 433 & " & Crucianella aegyptiaca L. & $\mathrm{Th}$ & Med. \\
\hline 434 & $"$ & Galium aparine $\mathrm{L}$. & Th & Med. \\
\hline 435 & $"$ & Galium murale L. & Th & Med. \\
\hline 436 & $"$ & Galium setaceum Lam. & $\mathrm{Th}$ & Med. \\
\hline 437 & $"$ & Galium tricornutum Dandy. & Th & Med. \\
\hline 438 & $"$ & Galium verrucosum Huds. & Th & Med. \\
\hline 439 & $"$ & Sheradia arvensis L. & $\mathrm{Th}$ & Med./ Ir-Tu. \\
\hline 440 & $"$ & Valantia hispida L. & Th & Med. \\
\hline 441 & $"$ & Valantia lanata Delile. & Th & Med. \\
\hline 442 & Rutaceae & Ruta chalepensis $\mathrm{L}$. & $\mathrm{Th}$ & Ir-Tu./ Sah-Ar. \\
\hline 443 & Santalaceae & Thesium bumile Vahl & $\mathrm{Th}$ & Med. \\
\hline 444 & Sapindaceae & Dodonea viscosa (L.) Jacq. & $\mathrm{NP}$ & Plu. \\
\hline 445 & Scrophulariacee & Kickxia egyptiaca L & $\mathrm{H}$ & Med./ Sah-Ar. \\
\hline 446 & " & Linaria simplex Desf. & Th & $\begin{array}{r}\text { Med./ Ir-Tu./ } \\
\text { Eur-Si. }\end{array}$ \\
\hline 447 & $"$ & Linaria tarbunensis Pamp. & $\mathrm{Th}$ & Med. \\
\hline 448 & $"$ & Linaria tenuis (Viv.) Sperng. & Th & Med./ Sah-Ar. \\
\hline 449 & $"$ & Misopates orontium L. \& Rafin. & Th & Med. \\
\hline 450 & $"$ & Scrophularia arguta Ait. & Th & Med./ Sah-Ar. \\
\hline 451 & Solanaceae & Lycium europaeum L. & NP & Med. \\
\hline 452 & " & $\begin{array}{r}\text { Lycium shawii Roemer \& } \\
\text { Schultes. }\end{array}$ & NP & Med./ Ir-Tu. \\
\hline 453 & $"$ & $\begin{array}{r}\text { Lycium showeinfurthii Dammer } \\
\text { in Bot. }\end{array}$ & NP & Med. \\
\hline 454 & $"$ & Nicotiana glauca R. C. Graham. & $\mathrm{NP}$ & Plu. \\
\hline 455 & $"$ & Solanum nigrum L. & Th & Cos. \\
\hline 456 & Tamaricaceae & Tamarix aphylla Graham. & $\mathrm{NP}$ & Sud./ Sah-Ar. \\
\hline 457 & Urticaceae & Perietaria mauritanica Durieu. & Th & Med. \\
\hline 458 & $"$ & Urtica pilulifera L. & $\mathrm{Th}$ & $\begin{array}{r}\text { Med./ Ir-Tu./ } \\
\text { Eur-Si }\end{array}$ \\
\hline 459 & $"$ & Urtica urens L. & $\mathrm{Th}$ & Med./ Ir-Tu. \\
\hline 460 & Valerianaceae & $\begin{array}{r}\text { Centranthus calcitrapa (L.) } \\
\text { Dufrense. }\end{array}$ & $\mathrm{Th}$ & Med. \\
\hline 461 & $"$ & Valerianella chlorodonata Cosson & Th & Med. \\
\hline 462 & " & Valerianella discoidea (L.) Loisel. & Th & Med./ Ir-Tu. \\
\hline 463 & " & Valerianella petrovichii Asherson. & Th & Med. \\
\hline 464 & Verbinaceae & Lantana camara $\mathrm{L}$. & NP & $\begin{array}{r}\text { Med./ Ir-Tu./ } \\
\text { Trop. }\end{array}$ \\
\hline 465 & Zygophyllaceae & Fagonia cretica L. & $\mathrm{H}$ & Med. \\
\hline 466 & & $\begin{array}{r}\text { Fagonia tenuifolia Steud. \& } \\
\text { Hochst. }\end{array}$ & $\mathrm{H}$ & Sah-Ar. \\
\hline 467 & $"$ & Peganum harmala L. & Th & Med./ Ir-Tu. \\
\hline 468 & $"$ & Tribulus terristris L. & Th & Plu. \\
\hline
\end{tabular}

